\title{
Erratum
}

\section{On the Uniqueness of Weak Weyl Representations of the Canonical Commutation Relation}

\author{
ASAO ARAI \\ Department of Mathematics, Hokkaido University, Sapporo 060-0810, Japan. \\ e-mail: arai@math.sci.hokudai.ac.jp
}

Published online: 19 May 2009 - (C) Springer 2009

\section{Erratum to: Lett Math Phys (2008) 85:15-25 DOI 10.1007/s11005-008-0252-9}

The statement on the complete reducibility of the $*$-algebra $\mathcal{W}_{+}$generated by $\{U(\alpha), V(\beta) \mid \alpha \geq 0, \beta \in \mathbb{R}\}$ in Remark 2.1 is inconclusive. Hence Remark 2.1 should be deleted. Corresponding to this change, the following corrections must be made (only essential points are indicated): (1) In Theorems 1.2, 1.3, 4.3 and Corollary 4.4, the irreducibility of the set $\mathcal{A}:=\left\{\bar{T}, T^{*}, H\right\}$, i.e., $\mathcal{A}^{\prime}$ (the strong commutant of $\mathcal{A})=\{c I \mid c \in \mathbb{C}\}$ should be assumed. (2) In Theorems 1.2 and 1.3, $N$ should be 1. (3) In Lemmas 2.4 and 2.5 , the irreducibility of $\mathcal{W}_{+}$should be assumed and $N$ should be 1. (4) In Examples 3.1 and 3.2, $d$ should be 1. (5) p. $23, \uparrow 1: " a \leq 0$ " $\rightarrow$ " $a=0$ " (the proof of Lemma 4.2 is not correct: one can show that $\operatorname{dim} \operatorname{ker}\left(d_{a}^{*}-i\right)=1$ for all $a<0$. (6) In Theorem 4.3 and in its proof, " $\varepsilon_{0} \leq 0$ " should be " $\varepsilon_{0}=0$ ". (7) In Corollary 4.4 , " $\mu \geq 0$ " should be " $\mu=0$ ".

The author would like to thank Professor L. Bracci, Professor L.E. Picasso and Y. Matsuzawa for their questions and comments.

The online version of the original article can be found under doi:10.1007/s11005-008-0252-9. 